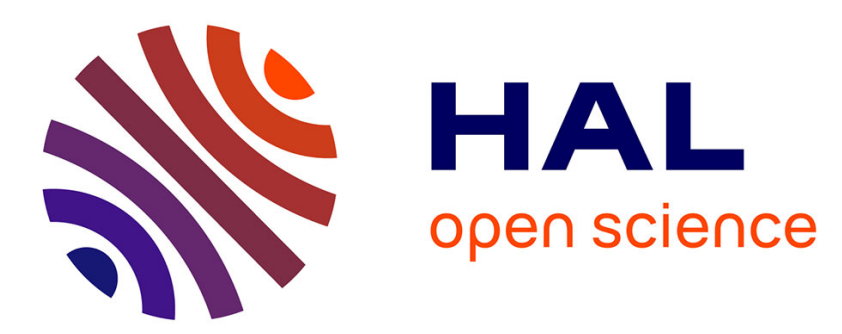

\title{
High performance computing of discrete nonsmooth contact dynamics with domain decomposition
}

\author{
Vincent Visseq, Pierre Alart, David Dureisseix
}

\section{To cite this version:}

Vincent Visseq, Pierre Alart, David Dureisseix. High performance computing of discrete nonsmooth contact dynamics with domain decomposition. International Journal for Numerical Methods in Engineering, 2013, 96 (9), pp.584-598. 10.1002/nme.4578 . hal-00918064

\section{HAL Id: hal-00918064 \\ https://hal.science/hal-00918064}

Submitted on 1 Nov 2016

HAL is a multi-disciplinary open access archive for the deposit and dissemination of scientific research documents, whether they are published or not. The documents may come from teaching and research institutions in France or abroad, or from public or private research centers.
L'archive ouverte pluridisciplinaire HAL, est destinée au dépôt et à la diffusion de documents scientifiques de niveau recherche, publiés ou non, émanant des établissements d'enseignement et de recherche français ou étrangers, des laboratoires publics ou privés. 


\title{
High performance computing of discrete nonsmooth contact dynamics with domain decomposition
}

\author{
Vincent Visseq ${ }^{1}$, Pierre Alart*1,3, and David Dureisseix ${ }^{2}$ \\ ${ }^{1}$ Laboratoire de Mécanique et Génie Civil (LMGC), Université \\ Montpellier 2, CNRS UMR 5508, F-34095 Montpellier CEDEX 5, \\ France \\ ${ }^{2}$ Laboratoire de Mécanique des Contacts et des Structures \\ (LaMCoS), Université de Lyon, INSA-Lyon, CNRS UMR 5259, \\ F-69621, Villeurbanne, France \\ ${ }^{3}$ Laboratoire de Micromécanique et d'Intégrité des Structures \\ (MIST), IRSN, CNRS, Université Montpellier 2, F-34095 \\ Montpellier CEDEX 5, France
}

\begin{abstract}
We investigate two algorithms for solving large-scale granular problems using domain decomposition methods. These numerical schemes can be connected to classical domain decompositions, with and without overlapping, developed in continuum mechanics. The two algorithms are compared in terms of implementation and parallel performance. We focus the numerical study on communication procedures between processors, load balancing and scalability, topics particularly crucial in nonlinear evolutive problems.

This is the accepted version of the following article: Visseq, V., Alart, P. and Dureisseix, D. (2013), High performance computing of discrete nonsmooth contact dynamics with domain decomposition. Int. J. Numer. Meth. Engng, 96: 584598. doi:10.1002/nme.4578, which has been published in final form at

http://onlinelibrary.wiley.com/doi/10.1002/nme.4578/abstract
\end{abstract}

Keywords: discrete elements; multibody; parallel computing; distributed memory; message passing; FETI method; additive Schwarz method; LMGC90; granular material

\section{Introduction}

Numerical simulations of granular media, at the grain scale, leads to several issues: non smoothness of frictional contact behavior (when no regularization

*Corresponding author, Pierre.Alart@univ-montp2.fr 
is used), multiplicity of solutions (for dense and a not so dynamical regime at macroscopic scale), and time consuming solvers (for large collections of grains and interactions). To address these issues, several answers had been proposed, for instance, several time stepping schemes [1,13], and parallelization of solvers $[16,27,28,29]$.

Here, we focus on non smooth contact dynamic (NSCD) method for the dynamical modeling and time stepping, and a domain decomposition method (DDM) as a support for distributed memory parallelization with message passing $[23,19]$. We discuss algorithms with two different proposals with a distribution of contact interactions among subdomains: a non overlapping FETI-like DDM, defining an interface gluing step thanks to the use of Lagrange multipliers, and a minimal overlapping Schwarz-like DDM with impulse coupling terms. In addition, two different implementations of the underlying communication scheme between processors are proposed. The numerical and parallel performances are compared and assessed on large scale 3D granular media simulations.

Up to our knowledge, the only solution methods coupling a domain decomposition method with non-regular implicit contact dynamics for granular models are available in $[5,23,29]$. The approach described in [23] mainly uses an asynchronous distributed-memory Non-Linear Gauss-Seidel solver (close to the one used in [17] for a synchronous solver and in [18] for an asynchronous version, both for shared-memory architectures. They correspond to the algebraic partitioning of the reduced dynamics, i.e. a splitting of grains between processors, while herein, we rely on a splitting of interactions between processors). Good parallel efficiencies are obtained in [23] with the use of a tuned dynamic load balancing, data migration, and duplication of the whole grain database among processors to minimize information exchanges. It illustrates the fact that there is still a scope for efficiency gains using a load-balancing algorithm. This tendency is confirmed in the similar approach of [29] for 2D problems addressing a large number of processors (up to 256). The distribution of interactions between processors has first been studied in [5] for a shared-memory architecture, smallsized 2D problems and the same kind of decomposition using a 'box method'; the common point with the Schwarz-NSCD method described in this article is the information exchange between processors, related to the grain global forces.

Section 2 briefly recalls the background theory of the nonsmooth contact dynamics (NSCD) in the context of time-stepping schemes. Section 3 focuses on domain decomposition for such a problem, and proposes several alternatives, that are compared on a first test example. Section 4 is related to the parallel performances for a large scale problem on a supercomputing architecture. Finally, Section 5 concludes and discusses outlooks of this work.

\section{Nonsmooth contact dynamics reference prob- lem}

With a time-stepping scheme on a dynamical analysis of a granular media, no event detection is performed. Once the solution is known at the beginning $t_{-}=t_{i}$ of a time slab $\left[t_{i}, t_{i+1}\right]$, whose known quantities are denoted with a 
superscript (-), the quantities at the end of the time slab (without a superscript) have to be determined.

\subsection{Grain nonsmooth dynamics}

Considering a rigid model, the dynamics of a grain over a time step is expressed with the vector equation [26]:

$$
M V-R=R^{d}
$$

where the prescribed right-hand side is $R^{d}=R^{D}+M V^{-}, V$ is the velocity of the grain (it contains the translational degrees of freedom (dof), and the rotational ones), while $R$ is the resultant impulse on the grain due to interactions with other grains and $R^{D}$ are the external prescribed impulses. The matrix $M$ contains both the mass (for the translational dof) and the inertia (for the rotational dof). A choice leading to a constant, and diagonal, matrix $M$ consists in expressing the global coordinates of rotation vectors in the inertia eigenbasis of each grain, see Figure 1. The assembly of these equations (independent for each grain) for all the involved grains in a granular medium is formally written in the same way as equation (1), which then represents the assembly of the individual grain equations.

\subsection{Contact interactions}

For a unilateral contact, Moreau proved via a viability lemma [26], that a velocity-impulse formulation may be equivalent to the classical complementary law between gap and contact force. It is then possible to admit velocity jumps and simultaneous contacts. The second interest is to handle, simultaneously, more complex interactions like friction, wear, adhesion, some of them often written with velocity. The interaction laws are not detailed in this paper. We simply summarize them in a formal relationship,

$$
\mathcal{R}(v, r)=0,
$$

between the relative velocity at the contact point between the two interacting grains, $v$, and the impulse at the same contact point, $r$. The relationship $\mathcal{R}$ is usually composed of nonlinear and multivalued mappings between the previous two dual quantities. The assembly of the interaction-related quantities for all interactions is also written formally in the same way (2). Both $v$ and $r$ are expressed in the local coordinate basis to the contacts between the interacting grains, see Figure 1. Therefore, they are linked to the global kinematic and static quantities using a compatibility condition, $H$ being the mapping matrix with a finite rotation:

$$
v=H^{T} V \quad \text { and } \quad R=H r
$$

\subsection{Reference problem and generic solver}

Taking the dynamic equation (1) and the compatibility conditions (3) into account, the reduced dynamics involving 'material' quantities can be obtained: 


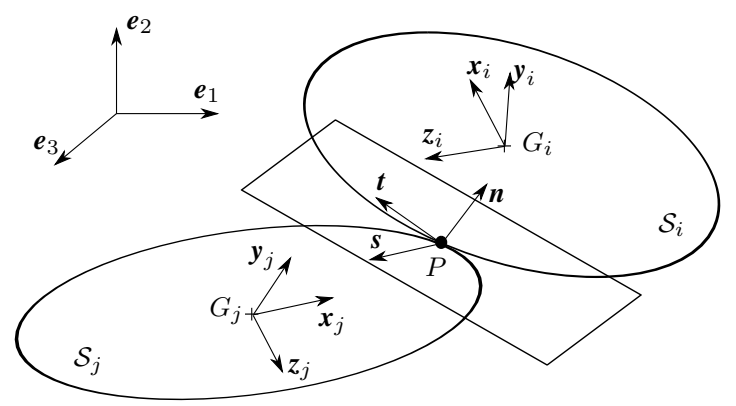

Figure 1: Coordinate basis; $R_{g}=\left(\boldsymbol{e}_{1}, \boldsymbol{e}_{2}, \boldsymbol{e}_{3}\right)$ : global coordinate basis (for interface quantities), $R_{i}=\left(\boldsymbol{x}_{i}, \boldsymbol{y}_{i}, \boldsymbol{z}_{i}\right)$ : local (related to the grain $\left.\mathcal{S}_{i}\right)$ coordinate basis (for grain dynamics), $R_{\alpha}=(\boldsymbol{n}, \boldsymbol{t}, \boldsymbol{s})$ : local (related to an interaction $\alpha$ between two grains) coordinate basis (for interactions). Details of the local contact frame $(\boldsymbol{n}, \boldsymbol{t}, \boldsymbol{s})$ at a contact $\alpha$ between two touching particles $\mathcal{S}_{i}$ and $\mathcal{S}_{j}$ at point $P$.

$$
W r-v=-v^{d}
$$

where $W$ is the Delassus operator, $W=H^{T} M^{-1} H$, and $v^{d}=H^{T} M^{-1} R^{d}$. To close the problem, one adds the constitutive relation (2), and the reference problem reads:

$$
\left\{\begin{array}{l}
W r-v=-v^{d} \\
\mathcal{R}(v, r)=0
\end{array}\right.
$$

The difficulty to solve this problem is at least two-folds: on one hand, the number of unknowns (number of interaction quantities $r$ and $v$ ) may be large (for instance, an average of $2 \times 10^{6}$ unknowns for the 3D problem illustrating this paper), and the Delassus operator $W$ is not well conditioned. On the other hand, the constitutive relation is nonsmooth (e.g. it is non linear, and not differentiable). To address the nonsmoothness issue, the NSCD (nonsmooth contact dynamics) method with a non-linear Gauss-Seidel (NLGS) solver [26, $20]$ is used. The convergence of such an algorithm is a difficult topic with few theoretical results [21] because of the multiplicity of solutions and the lack of regularity of the equations. Based on numerical experiments, the NLGS algorithm reveals to be the most robust solver that supports the more general interactions.

To address the large size of the problem, two substructuring approaches are proposed, as described below.

\section{Domain decomposition approaches}

Several domain decomposition approaches have been proposed for contact problems, see $[8,3,10,9,4,24,3]$ amongst others, but a few are concerned with multiple contacts in granular media (for an algebraic-like partition, see [17], and for a geometric partitioning of the discrete granular domain, see $[5,2,23,30]$ ). Here, we focus on the comparison of two formulations, one similar to FETI approaches, the other closer to additive Schwarz approaches, and of two implementations of the underlying communication scheme. 


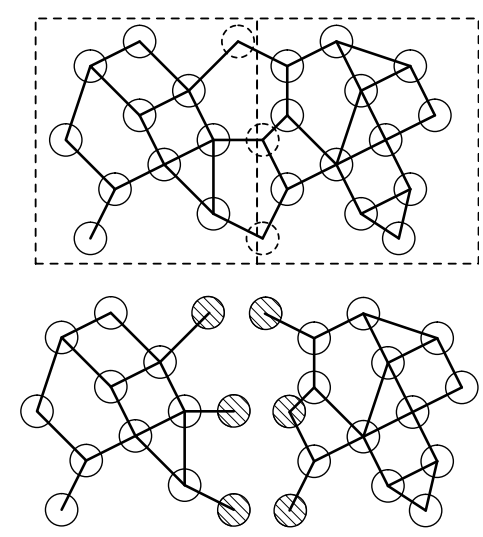

Figure 2: Geometrical partitioning of the discrete domain: inner grains are plotted empty and interface grains striped.

\subsection{Splitting choice and grain multiplicity}

The domain has to be split into subdomains in order to use parallel computing with data locality. This decomposition is performed as frequently as needed to take into account the migration of grains from one subdomain to another. Since the nonsmoothness may occur in interactions between grains, we choose to distribute interactions among subdomains as in [2] (we proceed by distributing the middle points between the centers of mass of interacting grains, according to their coordinates, using an arbitrary regular underlying grid, see Figure 2). If a grain indexed with $\mathcal{S}_{i}$ is connected with $m_{i}$ subdomains, $m_{i}$ is called its multiplicity number. The interface between two subdomains is defined as the set of these grains, that joins the subdomains. The nonsmoothness is therefore localized only within the subdomains. This modeling choice is identical to [4] and somehow the dual of that proposed in [24], where nonlinearities (contact on crack lips) are isolated at the interfaces. Note that a direct algebraic partitioning of the reference problem can also be chosen, leading to a dual partitioning and a different algorithm [17]. Some advantages and disadvantages have been discussed in previous works [17,30].

A grain is defined as belonging to (at least) one subdomain $E$ if it is connected to an interaction that belongs to this subdomain. Therefore, a boolean matrix $B_{E}$ selecting kinematic degrees of freedom of grains belonging to subdomain $E$ allows to define the grain velocities in this subdomain as:

$$
V_{E}=B_{E} V
$$

With this definition of the mapping matrix, one can check that the diagonal matrix of the grain multiplicities is $\sum_{E}^{n_{\mathrm{sd}}} B_{E}^{T} B_{E}$.

\subsection{FETI-like domain decomposition (NSCDD algorithm)}

This first DDM considers a non overlapping partition of the sample. For consistency with the rigid model of the grains, the masses and moments of inertia are distributed among the neighboring subdomains according to their multiplicity number. More precisely the distribution of masses and inertia is an algebraic 
partitioning and not a geometrical partitioning. For the elastic deformable model of the grains, this splitting can be performed with a classical mesh decomposer. This leads to a partition of unity over the inertia parameters, as:

$$
\tilde{M}_{E}=B_{E} D M B_{E}^{T}
$$

with:

$$
D_{k l}= \begin{cases}0 & \text { if } k \neq l \\ 1 / m_{i} & \text { if } k=l\end{cases}
$$

for entries $k$ related to the grain $\mathcal{S}_{i}$. The partition of unity property reads: $M=\sum_{E} B_{E}^{T} \tilde{M}_{E} B_{E}$.

This topic is investigated in details in [30]. In each subdomain $E$, the problem is identical to the global one (with the subscript $E$ ), provided that a term arising from the inter-grain interface is added. It can be built from the interconnecting condition (on the velocity jumps of boundary grains) that has been added to 'glue' neighboring subdomains, where $A_{\Gamma E}$ is a signed boolean matrix with a finite rotation, to map the grain velocities $V_{E}$ to the global coordinate basis into which the null velocity jump on the grain interface is expressed:

$$
\sum_{E=1}^{n_{\mathrm{sd}}} A_{\Gamma E} V_{E}=0
$$

$\Gamma$ denotes the global interface of all the interface grains. Formally the previous summation is performed on all the subdomains (whose number is equal to $n_{\mathrm{sd}}$ ); in a practical way, for a given grain interface, only the neighboring subdomains have to be considered. We then obtain a FETI-like formulation [12] for the reference problem using a multiplier field $F_{\Gamma}$ and the notation $\hat{A}_{\Gamma E}^{T}=H_{E}^{T} \tilde{M}_{E}^{-1} A_{\Gamma E}^{T}$, $\tilde{W}_{E}=H_{E}^{T} \tilde{M}_{E}^{-1} H_{E}$ :

$$
\left.\begin{array}{l}
\tilde{W}_{E} r_{E}-v_{E}-\hat{A}_{\Gamma E}^{T} F_{\Gamma}=-v_{E}^{d} \\
\mathcal{R}\left(v_{E}, r_{E}\right)=0 \\
\sum_{E=1}^{n_{\mathrm{sd}}} A_{\Gamma E} V_{E}=0
\end{array}\right\} E=1, \ldots, n_{\mathrm{sd}}
$$

The reduced problem on $\left(r_{E}, v_{E}, F_{\Gamma}\right)$, with the notations $\hat{f}=\sum_{E} A_{\Gamma E} \tilde{M}_{E}^{-1} R_{E}^{d}$, $v_{E}^{d}=H_{E}^{T} \tilde{M}_{E}^{-1} R_{E}^{d}$ and $X=\sum_{E} A_{\Gamma E} \tilde{M}_{E}^{-1} A_{\Gamma E}^{T}$ and a partial condensation of the problem, reads:

$$
\left.\begin{array}{l}
\tilde{W}_{E} r_{E}-v_{E}-\hat{A}_{\Gamma E}^{T} F_{\Gamma}=-v_{E}^{d} \\
\mathcal{R}\left(v_{E}, r_{E}\right)=0 \\
X F_{\Gamma}-\sum_{E=1}^{n_{\mathrm{sd}}} \hat{A}_{\Gamma E} r_{E}=\hat{f}
\end{array}\right\} E=1, \ldots, n_{\mathrm{sd}}
$$

One easily reformulates the interface equation as an incremental problem [30]: if $F_{\Gamma}$ is associated to a velocity field $V$ with the dynamical equations, and if this last field is not continuous at the interface, the correction of the impulse field $F_{\Gamma}$ is $\Delta F_{\Gamma}$ such that

$$
X \Delta F_{\Gamma}=\sum_{E=1}^{n_{\mathrm{sd}}} A_{\Gamma E} V_{E}=[V]_{\mid \Gamma}
$$


the last term being the residual on the interface, i.e. the velocity jump $[V]_{\mid \Gamma}$. As for many domain decomposition approaches, the goal is to be able to localize the same typical problem that is under consideration on each subdomain independently, while designing a suited coupling recovery algorithm between subdomains, i.e. on the interface.

Here, the algorithmic formulation described in Algorithm 1 has been implemented into the LMGC90 platform [11] for time-evolution problems $(N$ is the number of time steps). At each new time step of the incremental solving procedure, the mapping $H$ and the contact graph have to be updated within a contact detection phase. Eventually, the domain could also be repartitioned according to the new contact graph.

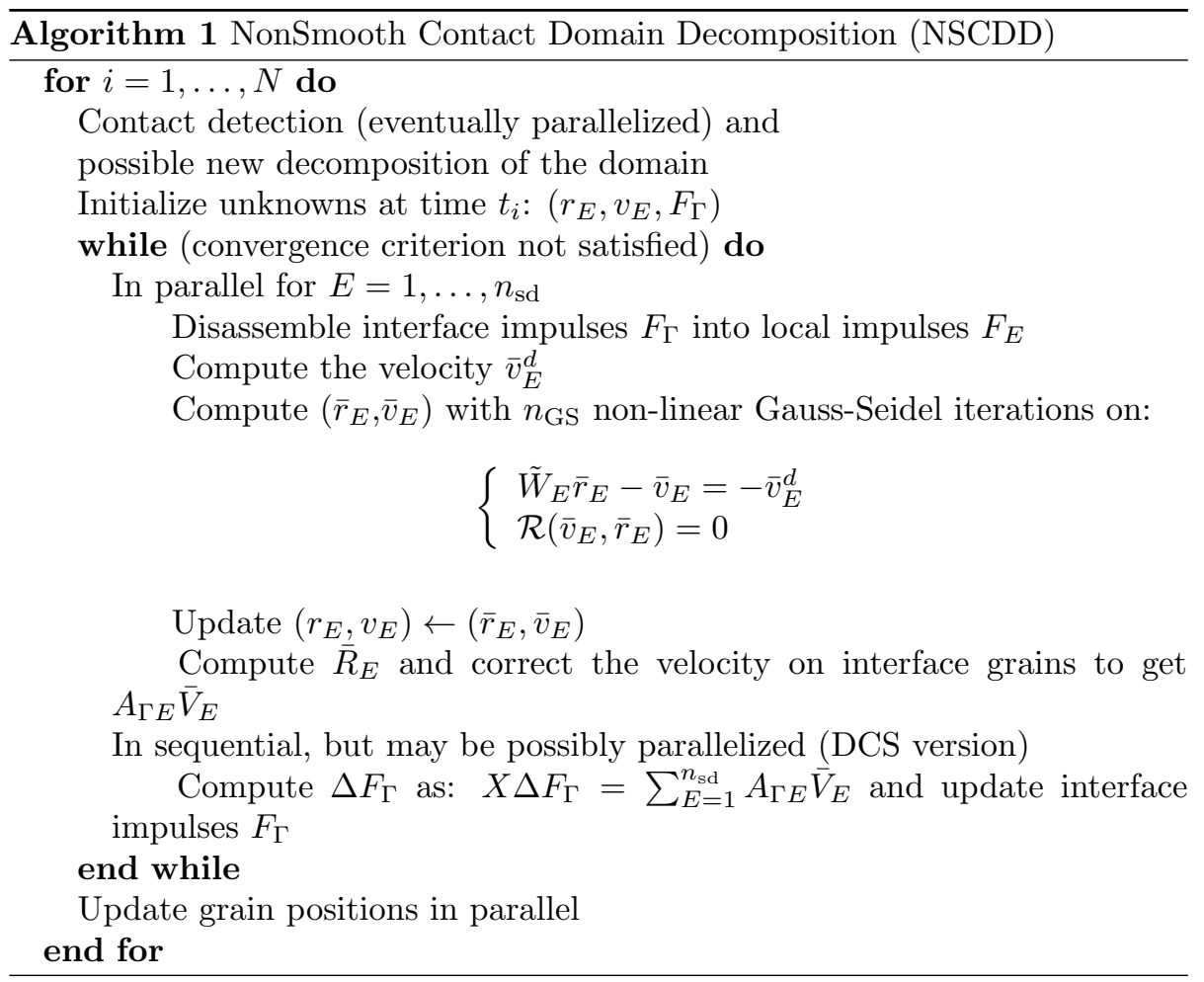

\subsection{NSCDD interface solving procedures}

Centralized communication scheme (CCS). At first glance, the interface gluing step (12) is defined as a global linear equation linking all the subdomains. It requires communications between the subdomains such that one process gathers all the velocity contributions to the vector of velocity jumps $[V]_{\mid \Gamma}$. The value of the Lagrange multipliers $F_{\Gamma}$ computed sequentially is then distributed such that subdomain $E$ receives its minimal required data amount $A_{\Gamma E}^{T} F_{\Gamma}$.

Decentralized communication scheme (DCS). Due to the structure of the interface operator $X$, extensively studied in [30], each distributed database 


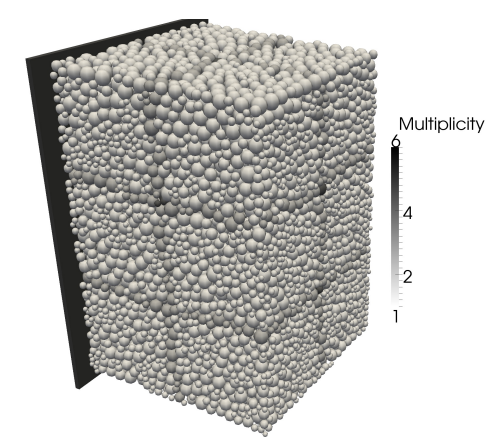

Figure 3: A rigid wall supporting contacts in a long range (a whole side of the 16000 spheres sample partitioned in twelve subdomains), is involved in the local interface of six subdomains.

(per process related to subdomain $E^{*}$ ) is sufficient to get the elementary contribution to the interface operator:

$$
X_{\Gamma_{E^{*}}}=\sum_{E=1}^{n_{\mathrm{sd}}} A_{\Gamma_{E^{*}} E} \tilde{M}_{E}^{-1} A_{\Gamma_{E^{*}}}^{T}
$$

$A_{\Gamma_{E^{*}} E}$ is a signed boolean matrix, mapping grains of subdomain $E$ to velocity jumps of the elementary interface $\Gamma_{E^{*}}$ (restriction of the global interface to the boundary of subdomain $E^{*}$ ). Then, an elementary interface problem can be defined as:

$$
X_{\Gamma_{E^{*}}} \Delta F_{\Gamma_{E^{*}}}=\sum_{E=1}^{n_{\mathrm{sd}}} A_{\Gamma_{E^{*}} E} V_{E}
$$

Finally, the data gathering of $\sum_{E=1}^{n_{\mathrm{sd}}} A_{\Gamma_{E^{*}} E} V_{E}$ on each process corresponds to data exchanges over an unstructured topology. That means the exchanges do not concern only the subdomains which are geometrically neighbors, since some large/elongated rigid bodies may go through several subdomains. A common example of such a situation is a granular medium confined by rigid walls as illustrated in Figure 3. A wall performs a connection and exchanges between several non-neighboring subdomains. With the computation of the assembling of local contributions, it is easy to show that this is the expected iterated vector:

$$
\Delta F_{\Gamma}=\sum_{E=1}^{n_{\mathrm{sd}}} B_{\Gamma E} D_{E} B_{\Gamma_{E} E}^{T} \Delta F_{\Gamma_{E}}
$$

$B_{\Gamma E}$ is a boolean matrix selecting interface grains among subdomains, $B_{\Gamma_{E} E}$ is a boolean matrix selecting elementary interface grains among subdomains and $D_{E}$ is a diagonal matrix with value $1 / m_{i}$ for entries corresponding to grain $i$.

\subsection{Schwarz-like domain decomposition}

We define a Schwarz-like domain decomposition method based on fundamental properties of rigid body dynamics dealing with diffuse contact network. Considering the selecting operator $B_{E}$, directly derived from partitioning strategy 
defined in section 3.1 in (6), dynamic equations (1) per subdomain, i.e. for all the grains within a given subdomain, read:

$$
B_{E}(M V)=B_{E}\left(R+R^{d}\right)
$$

Considering an overlapping between subdomains, the restricted inertia matrix to a subdomain $E$ can be defined as,

$$
M_{E}=B_{E} M B_{E}^{T}
$$

The overlapping is rendered by the lack of partition of the unity in (17) in comparison with (7). Here the domain partitioning defines a minimal overlapping between subdomains, since the dynamical operators $M_{E}$, for $E=1, \ldots, n_{\text {sd }}$ duplicate the contribution of the interface grains. Then the left hand side reads:

$$
B_{E}(M V)=M_{E} V_{E}
$$

In a similar way, the given impulse $R^{d}$, which comes from velocity at the beginning of the time step and the external forces acting on grains, depends only on inner quantities of subdomain $E$ :

$$
B_{E} R^{d}=R_{E}^{d}
$$

As an interface grain supports contacts in more than one subdomain the coupling term between subdomains arises from contact impulses as:

$$
R=\sum_{E^{*}=1}^{n_{\mathrm{sd}}} B_{E^{*}}^{T} H_{E^{*}} r_{E^{*}}
$$

When extracting resultant impulse quantities according to $B_{E}, B_{E} R$ splits into internal and coupling terms such that:

$$
B_{E} R=R_{E}+R_{E}^{d d m} \quad \text { with } \quad R_{E}=H_{E} r_{E}
$$

and

$$
R_{E}^{d d m}=\sum_{E^{*} \neq E} B_{E} B_{E^{*}}^{T} R_{E^{*}}
$$

where $B_{E} B_{E^{*}}^{T}$ defines a boolean matrix whose non-zero entries correspond to grains from subdomain $E^{*}$ belonging also to subdomain $E$.

The dynamics of the granular medium per subdomain is therefore expressed with the vector equation:

$$
M_{E} V_{E}-H_{E} r_{E}=R_{E}^{d}+R_{E}^{d d m}
$$

and the reduced problem, with $v_{E}^{d d m}=H_{E}^{T} M_{E}^{-1} R_{E}^{d d m}$, reads:

$$
\left.\begin{array}{l}
W_{E} r_{E}-v_{E}=-v_{E}^{d}-v_{E}^{d d m} \\
\mathcal{R}\left(v_{E}, r_{E}\right)=0
\end{array}\right\} E=1, \ldots, n_{\mathrm{sd}}
$$

The algorithmic formulation of this method (see Algorithm 2) does not define the interface behavior but only data exchanges between subdomains over an unstructured topology (as for the NSCDD decentralized communication scheme). This differs also from the dual partitioning developed in [17], for which a non 
smooth problem similar to (24) is defined on the global interface composed of contacts, as a standard subdomain. Impulses acting on the boundary grains of a subdomain related to contacts belonging to interconnected subdomains are then taken as boundary conditions without defining a global interface problem as in the NSCDD method. This structure of DDM algorithm has the flavor of Schwarz algorithms [25, 6, 14]. This method will be called Schwarz-NSCD in the following.

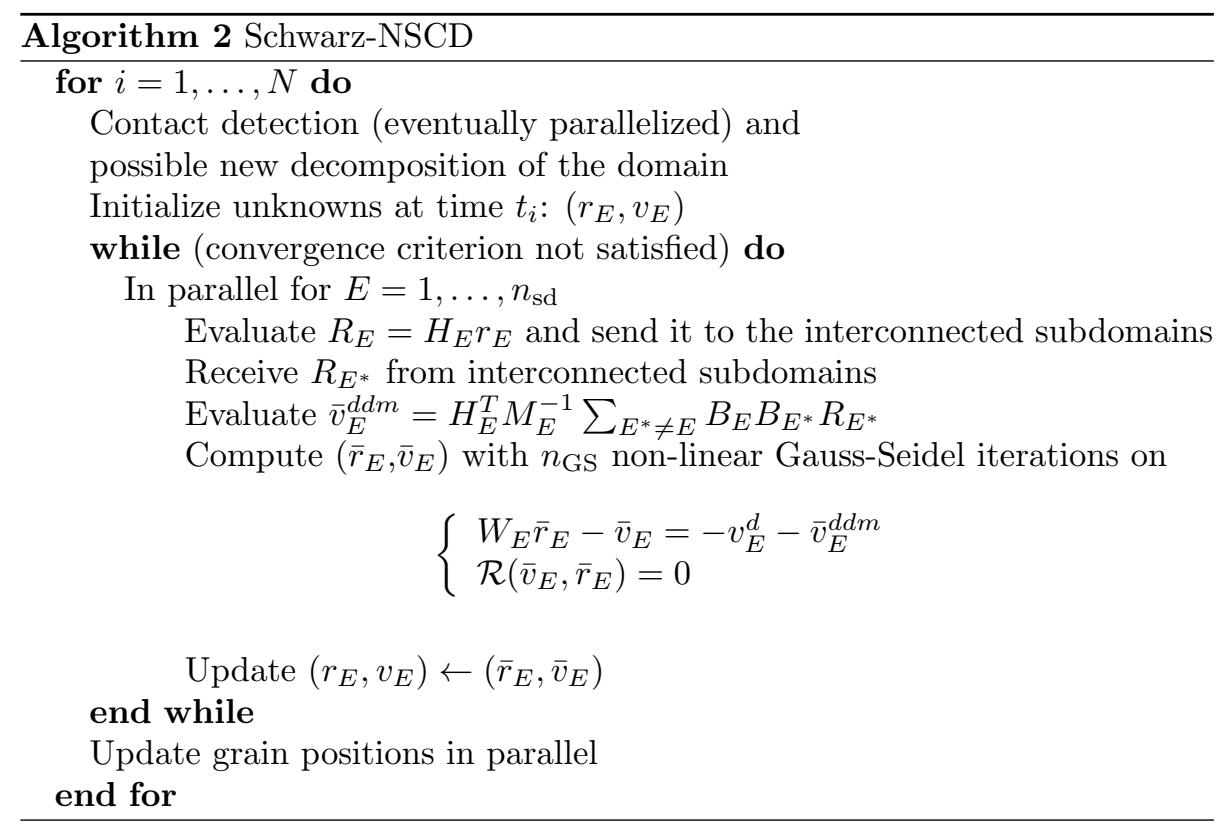

Algorithm 2 may be interpreted as a Gauss-Seidel-like method with a special numbering. Indeed, choosing $\alpha, \beta$ as indexes of contacts belonging to subdomain $E$, the Non Linear Gauss-Seidel $k+1$ iteration for contact $\alpha$ reads:

$$
\left\{\begin{array}{l}
W_{\alpha \alpha} r_{\alpha}^{k+1}-v_{\alpha}^{k+1}=-v_{\alpha}^{d}-\bar{v}_{\alpha}^{d d m}-\sum_{\beta<\alpha} W_{\alpha \beta} r_{\beta}^{k+1}-\sum_{\beta>\alpha} W_{\alpha \beta} r_{\beta}^{k} \\
\mathcal{R}\left(r_{\alpha}^{k+1}, v_{\alpha}^{k+1}\right)=0
\end{array}\right.
$$

If $i$ is the index (in the global numbering of the sample) of the interface grain associated to a contact $\alpha$, and $\gamma_{E *}$ the indexes of contacts of subdomain $E^{*}$ interconnected with $E$ via the grain $i$, and choosing $n_{\mathrm{GS}}=1, \bar{v}_{\alpha}^{d d m}$ reads:

$$
\begin{aligned}
\bar{v}_{\alpha}^{d d m} & =H_{\alpha}^{T} M_{i}^{-1} \sum_{E^{*} \neq E} R_{E^{*}}^{i} \\
& =H_{\alpha}^{T} M_{i}^{-1} \sum_{E^{*} \neq E} \sum_{\gamma_{E^{*}}} H_{\gamma_{E^{*}}} r_{\gamma_{E^{*}}}^{k} \\
& =\sum_{E^{*} \neq E} \sum_{\gamma_{E^{*}}} W_{\alpha \gamma_{E^{*}}} r_{\gamma_{E^{*}}}^{k}
\end{aligned}
$$

The choice $n_{\mathrm{GS}}=1$ means that the interface exchanges are performed after each Gauss-Seidel iteration; this is justified in previous works [2] for iterative schemes with robust but slow convergence. Rewriting equations (25) using the 
Table 1: Comparison of elapsed CPU time percentage consumed during MPI exchanges for centralized (NSCDD-CCS) and decentralized (NSCDD-DCS and Schwarz-NSCD) communication schemes; isotropic compaction of a 16000 spheres sample.

\begin{tabular}{cccc}
\hline$n_{\text {sd }}$ & Partitioning grid $\left(n_{x} \times n_{y} \times n_{z}\right)$ & NSCDD-CCS & NSCDD-DCS/Schwarz-NSCD \\
\hline 2 & $1 \times 1 \times 2$ & $6 \%$ & $2 \%$ \\
4 & $1 \times 2 \times 2$ & $25 \%$ & $3 \%$ \\
8 & $2 \times 2 \times 2$ & $47 \%$ & $9 \%$ \\
12 & $2 \times 2 \times 3$ & $57 \%$ & $10 \%$ \\
16 & $2 \times 2 \times 4$ & $69 \%$ & $16 \%$ \\
\hline
\end{tabular}
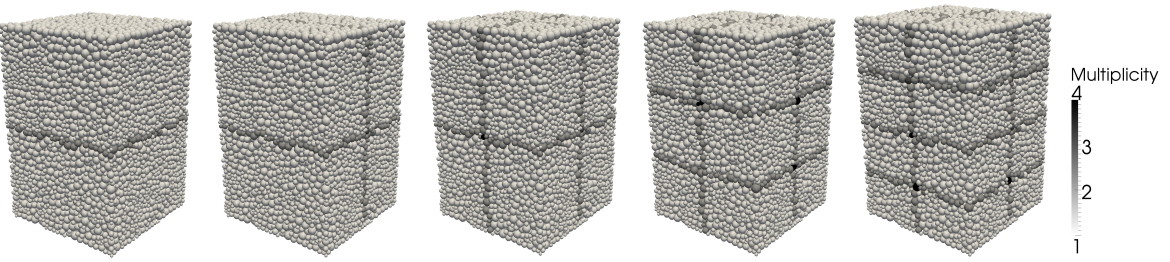

Figure 4: Sample of 16000 spheres submitted to isotropic compaction; tested decompositions to study the time spent in communications depending on the communication scheme. For convenience walls are not plotted on these figures.

expression of $\bar{v}_{\alpha}^{d d m}$ from equation (26) one obtains:

$\left\{\begin{array}{l}W_{\alpha \alpha} r_{\alpha}^{k+1}-v_{\alpha}^{k+1}=-v_{\alpha}^{d}-\sum_{\beta<\alpha} W_{\alpha \beta} r_{\beta}^{k+1}-\sum_{\beta>\alpha} W_{\alpha \beta} r_{\beta}^{k}-\sum_{E^{*} \neq E} \sum_{\gamma_{E^{*}}} W_{\alpha \gamma_{E^{*}}} r_{\gamma_{E^{*}}}^{k} \\ \mathcal{R}\left(r_{\alpha}^{k+1}, v_{\alpha}^{k+1}\right)=0\end{array}\right.$

These last equations reveal an hybrid Non linear Gauss-Seidel/Jacobi algorithm for the calculus of $\left(\bar{r}_{E}, \bar{v}_{E}\right)$ since the last summation relates only to values of $r_{\gamma_{E^{*}}}$ at the previous iteration.

\subsection{Comparison of the different approaches}

The influence of the proposed communication schemes is studied regarding the CPU time percentage consumed during MPI (Message Passing Interface [15]) exchanges (Table 1) with respect to the whole CPU time of a simulation. The test consists of a sample with 16000 spheres submitted to an isotropic compaction, over 500 time steps (Figure 4). Increasing the number of subdomains with a fixed problem size leads to increase the interface size and to reduce the local problem size on each subdomain.

Results (obtained on the supercomputer HPC@LR) presented in Table 1 show clearly the gain we may obtain considering DCS compared to CCS. Decentralized communication scheme (similar for NSCDD-DCS or Schwarz-NSCD) decreases drastically the time consumed in MPI exchanges in comparison to centralized one, such that time percentage consumed in exchanges between processors stays quite small for a reasonable domain partitioning. An heuristic rule for $3 \mathrm{D}$ dense granular material may be to chose a domain partitioning such that each domain contains at least $10^{3}$ grains. 


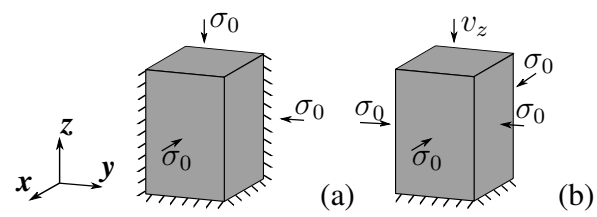

Figure 5: Isotropic compaction (a) and triaxial loading (b)

In the following we choose to study the numerical performances of the only NSCDD algorithm, with decentralized communication scheme, for a large granular sample.

\section{Performances and scalability results}

\subsection{Numerical example}

Sample preparation. A dense packing composed of $2 \times 10^{5}$ spheres is prepared in two phases. First a layer-by-layer deposition model based on simple geometrical rules is performed [31]. The particles are deposited sequentially on a substrate. Each new particle is placed at the lowest possible position on the free surface as a function of its diameter. This procedure leads to a random packing in which each particle supports at least three contacts. To avoid long range ordering a small polydispersity in size is used.

Once performed this geometrical process, the second phase is a compaction consisting of an isotropic compression inside a prismatic frame of dimensions $L_{0} \times l_{0} \times h_{0}$ in which three walls are fixed, and three walls are subjected to a compressive stress $\sigma_{0}$. The gravity $g$ and friction coefficients $\mu$ between the particles and the walls are set to zero during the compression in order to avoid force gradients and obtain an isotropic dense packing, see Figure 5(a).

Triaxial test. The isotropic sample is then subjected to a vertical compression by downward displacement of the top wall with a constant velocity $v_{z}$ for a constant confining stress $\sigma_{0}$ acting on the lateral walls, see Figure $5(\mathrm{~b})$. The friction coefficient $\mu$ between particles is set to 0.35 and to zero with the walls. Since we are interested in a quasistatic macroscopic behavior, the shear rate should be such that the kinetic energy supplied by shearing is negligible compared to the static pressure. The cumulative strain components $\varepsilon_{\lambda}$ are defined as:

$$
\varepsilon_{\lambda}=\int_{\lambda_{0}}^{\lambda} \frac{d \lambda^{\prime}}{\lambda^{\prime}}=\ln \left(1+\frac{\Delta \lambda}{\lambda_{0}}\right), \quad \lambda \in\{h, L, l\}
$$

where $h_{0}, L_{0}$ and $l_{0}$ are the initial height, width and length of the simulation box, respectively, and $\Delta h=h-h_{0}, \Delta L=L-L_{0}$ and $\Delta l=l-l_{0}$ are the corresponding cumulative displacements. The volumetric strain reads:

$$
\varepsilon_{V}=\int_{V_{0}}^{V} \frac{d V^{\prime}}{V^{\prime}}=\ln \left(1+\frac{\Delta V}{V_{0}}\right)
$$




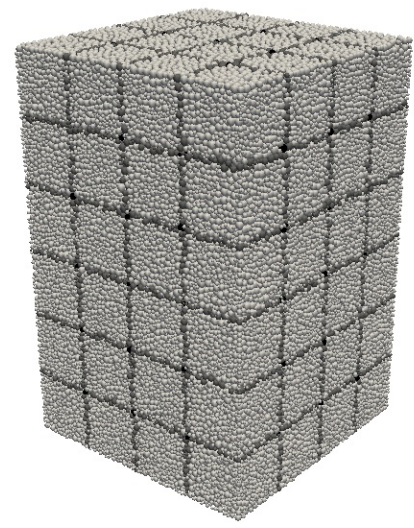

(a)

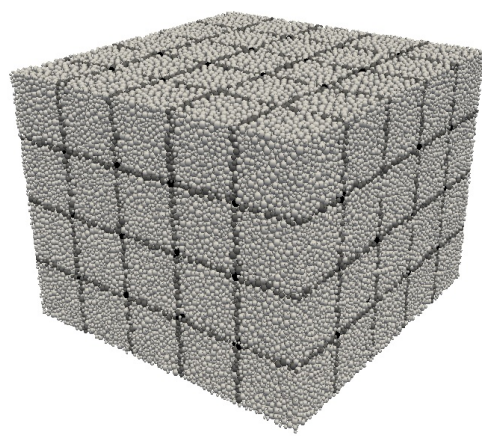

(b)

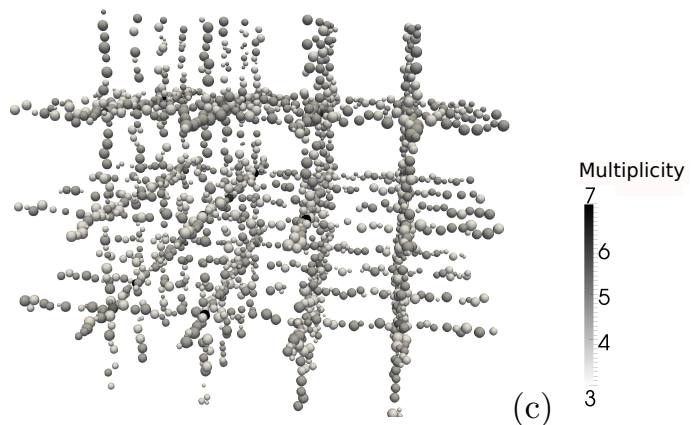

Figure 6: Domain partitioning of the sample at $\varepsilon_{V}=0$ (a) and $\varepsilon_{V}=0.7$ (b) and underlying structure of corner grains at $\varepsilon_{V}=0.7$ (c). For convenience (a) and (b) gray scale is restricted to $[1,4]$ and (c) gray scale is restricted to $[3,7]$; $m=1$ : inner grain; $m=2$ : face grain; $m>2$ : corner grain. The configuration (b) is split into 100 subdomains in order to adapt the partitioning to the aspect ratio.

where $V_{0}$ is the initial volume and $\Delta V=V-V_{0}$ is the total volume change. The cumulative shear strain is defined as:

$$
\varepsilon_{V} \equiv \varepsilon_{h}-\varepsilon_{l}
$$

The solid fraction $\rho$ is defined by $\rho=V_{p} / V$, with $V_{p}$ the volume occupied by particles.

Mechanical validation. The simulation is performed using the domain partitioning depicted in Figure 6 up to $\varepsilon_{V}=0.65$. The domain partitioning and the NSCDD algorithm does disturb neither the macroscopic behavior nor the micromechanical statistical analysis of the granular medium, as shown in [30], whereas increasing the number of particles allows to avoid boundary effects and improves the statistical analysis one can perform. Due to the indeterminacy of the problem, we monitored both the quality of the solution obtained considering classical convergence criteria, into each subdomain [7] and over the interface [19], and the mean/max interpenetration quantities: mean interpenetration remained below $0.02 \%$ of the min radius of the sample and maximum interpenetration remained below $10 \%$ of the minimum radius of the sample. Figure 7 depicts one 


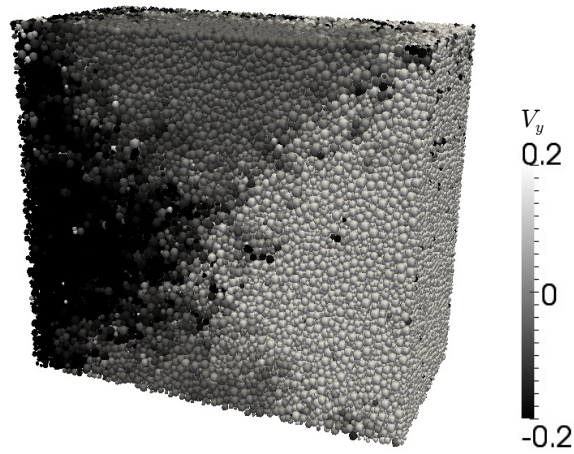

Figure 7: Velocity component along y axis at $\varepsilon_{V}=0.5$

map of the particles velocities at $\varepsilon_{V}$ over a slice of the sample. We see clearly the topology of the shear bands associated to localization phenomena. Figure 8 shows the contact network we deal with, composed of more than $2 \times 10^{6}$ force components.

\subsection{Scalability in nonsmooth evolutive problems}

We choose to quantify the parallel performances of the NSCDD algorithm by the usual quantities: the speedup $S_{p}$, which is the ratio of the run time of the non-parallel version on a single processor to the run time of the parallel version on $N_{p}$ processors and the efficiency $E_{p}=S_{p} / N_{p}$. Results are obtained setting the parameter $n_{\mathrm{GS}}$ equals to 1 and for 3 typical states of the sample during its evolution. Complementary informations on those states are given in Table 2.

- "Loose state" ( $\rho=0.567)$ occurs in the preparation phase which is an essential stage for providing an isotropic sample. More precisely it is obtained by deposit routines, at the beginning of the isotropic compaction phase. This state is characterized by a relatively small number of active contacts in comparison with the number of particles. Moreover a frictionless contact law leads to a quick convergence; the reduced dynamic problem is therefore quite cheap to solve. The goal is to provide a random close packing (RCP) sample.

- "RCP state" ( $\rho=0.648$ in our case) is used to start triaxial test, where the number of contacts is maximal. Due to confinement, the number of NSCDD iterations is large because of the indeterminacy of the problem amplified by the friction.

- "Residual state" (from $\rho=0.617$ to $\rho=0.612$ in our case) corresponds to a plateau of the shear stress evolution for which the shear bands appearance in the sample implies the decrease of the number of contacts in comparison with the RCP state.

Figure 9 depicts evolution of the speedup as a function of the number of processors (equal to the number of subdomains). Results obtained strongly depend on the state of the granular material. For the "loose state" the speedup diverges rapidly from the linear graph with slope equal to 1 . Instead that, for 


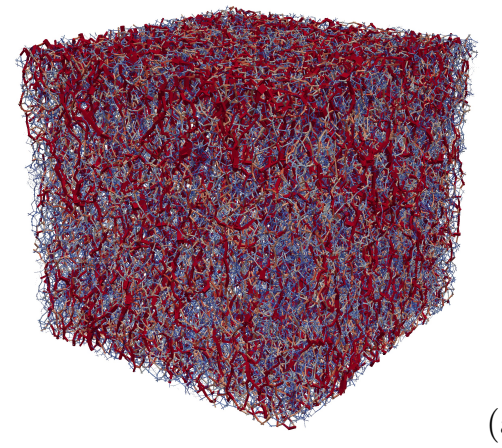

(a)
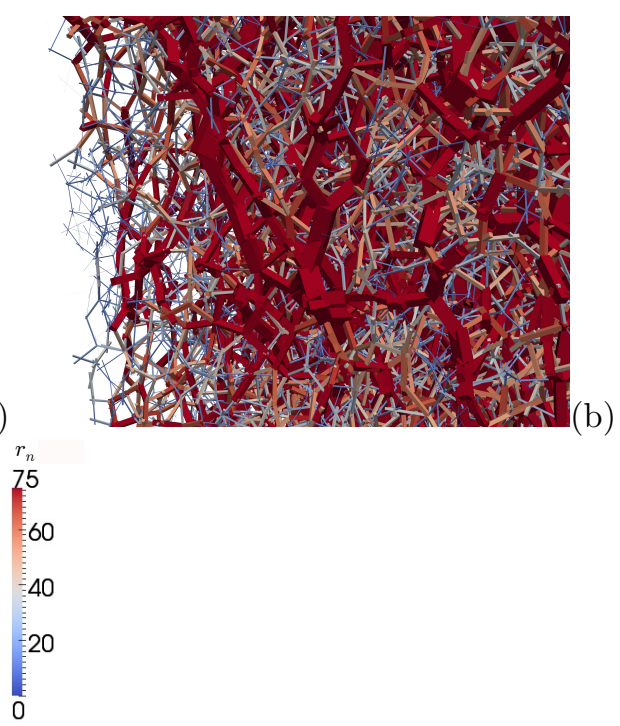

Figure 8: Force contact network at $\varepsilon_{V}=0.5$; full sample (a) and zoom (b). Only normal components are depicted.

Table 2: Main characteristic values of the 3 states used to study the speedup behavior of the simulations; $N_{C}$ is the mean number of contacts per time step, $I t_{\mathrm{NSCDD}}$ is the mean number of NSCDD iterations per time step and $N_{\mathrm{MG}}$ is the mean number of migrating grains per time step (i.e. whose subdomain is changing).

\begin{tabular}{cccc}
\hline State & $N_{C}$ & $I t_{\mathrm{NSCDD}}$ & $N_{\mathrm{MG}}$ \\
\hline "Loose" & 339957 & 701 & 38 \\
"RCP" & 669665 & 11978 & 24 \\
"Residual" & 567957 & 9993 & 29 \\
\hline
\end{tabular}



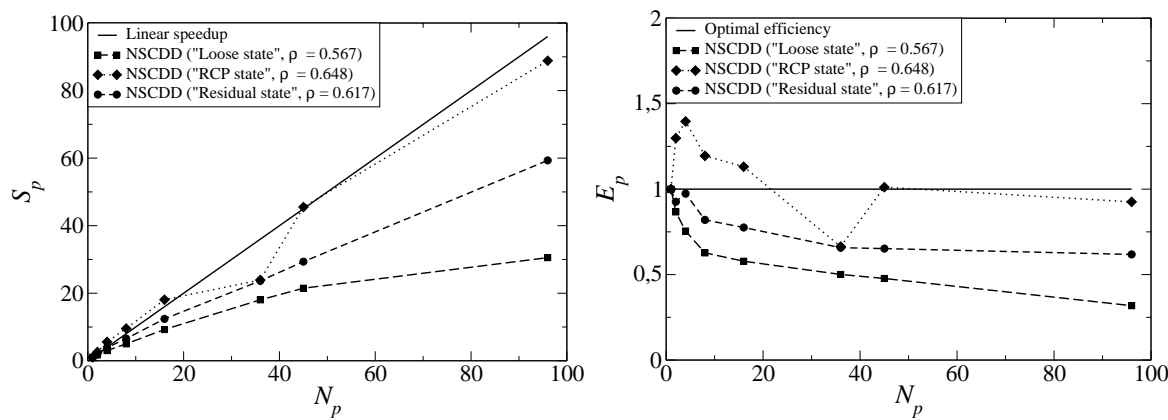

Figure 9: Speedup and efficiency as functions of the number of processors.

Table 3: Time consuming percentage of the main steps of the NSCDD algorithm; Partitioning stands for the data gathering, sequential routines of partitioning of the sample, scattering of local interface informations and treatment of migrating grains; Contact detection relates to the parallel construction of $H_{E}$ operators; Inloop 1 stands for parallel calculus routines for iterative solving of the reduced dynamics (Gauss-Seidel iterations and interface updating); Inloop 2 stands for MPI exchanges during the iterative solving of the reduced dynamics; Update stands for parallel calculus routines for updating velocities and generalized configuration of grains.

\begin{tabular}{cccccc}
\hline State & Partitioning & Contact detection & Inloop 1 & Inloop 2 & Update \\
\hline "Loose" & $11.0 \%$ & $2.6 \%$ & $66.7 \%$ & $10.7 \%$ & $9 \%$ \\
"RCP" & $4.4 \%$ & $0.7 \%$ & $76.7 \%$ & $15.6 \%$ & $3.9 \%$ \\
"Residual" & $2.0 \%$ & $0.2 \%$ & $82.1 \%$ & $12.5 \%$ & $3.2 \%$ \\
\hline
\end{tabular}

the "RCP state" and the "Residual state", the speedup behavior is almost linear but not with the same slope (apart from one value related to the "RCP state" for 36 processors which illustrates variability phenomenon when using computer center). Speedup culminates to a value of 88 for 96 subdomains in "RCP state". This shows that the NSCDD method is particularly relevant for dense granular material.

Table 3 shows that the three analyzed states differ as regards to the repartition, among the main stages of the algorithm, of CPU elapsed time percentage. Strong differences appear for "loose state" compared to the two others: partitioning stage becomes non negligible, as regards to solving procedure of reduced dynamics, whereas the number of contacts $N_{C}$ and the NSCDD iterations $I t_{\mathrm{NSCDD}}$ are smaller and the number of migrating grains is larger (see Table 2). In all cases the partitioning stage is re-done every 10 time steps to detect migrating grains. More precisely a grain is declared migrant when it supports contacts in a subdomain where it did not stand previously.

Table 4 shows that the geometric partitioning proposed, based on a regular underlying grid, does not assure a perfect load balancing. Indeed the number of bodies belonging to a subdomain varies up to $23 \%$. For interface quantities this phenomena is even more present, as the number of interface grains per subdomain $N_{E}^{m>2}$ and the number of interconnected subdomains for a given subdomain $N_{E}^{E \text { Isd }}$ vary up to $73.8 \%$ and $91.4 \%$ respectively. This implies strong 
Table 4: Characteristic values per subdomains ("RCP state"); $N_{E}^{\mathcal{S}}$ stands for the number of grains belonging to subdomain $E ; N_{E}^{C}$ stands for the number of contacts; $N_{E}^{\text {Isd }}$ stands for the number of subdomains interconnected with a considered subdomain $E ; N_{E}^{m>2}$ stands for the number of corners grains; $T_{\mathrm{MPI}}$ Exch stands for the percentage of MPI exchange time consumed when solving reduced dynamics; minimum, maximum and variation percentage.

\begin{tabular}{lrrr}
\hline & Min & Max & Variation percentage \\
\hline$N_{E}^{S}$ & 2086 & 2633 & $23.2 \%$ \\
$N_{E}^{C}$ & 6514 & 6795 & $4.2 \%$ \\
$N_{E}^{\text {Isd }}$ & 20 & 52 & $91.4 \%$ \\
$N_{E}^{m}>2$ & 445 & 965 & $73.8 \%$ \\
$T_{\text {MPI Exch }}$ & $3.84 \%$ & $26.7 \%$ & $149 \%$ \\
\hline
\end{tabular}

fluctuations on the CPU time percentage consumed in MPI exchanges during the iterative process for solving non smooth dynamics. Nevertheless, the contacts, which are the main unknowns of dense granular problems, are well distributed among subdomains. Obtaining a good load balancing for both grains and contacts is quite a hard topic when considering a simple geometric partitioning but may be attempted using automatic graph partitioner such as ParMETIS [22], or other approaches such as orthogonal recursive bisection that proved their efficiency in [23, 29].

\section{Conclusions}

This article focused on numerical and parallel performances of a domain decomposition method suited to granular dynamics analysis. Two versions, namely a FETI-like approach and a Schwarz-like approach, have been implemented into the LMGC90 software platform and tested on typical 3D simulations of granular dynamics, and on a modern supercomputing hardware platform. Even without a dedicated load balancing, the speedup of the simulations exhibits good parallel behavior, for large scale granular systems. To do so, both contact detection and solver have been parallelized, up to 100 processors used for a problem with an average of $2 \times 10^{6}$ frictional contact interaction unknowns.

Next step will consist in a dynamic load balancing strategy, with a distributed database dynamical update to improve efficiency. On the other hand, additional strategies may decrease the computational cost by model adaptation and homogenization, to tackle even larger simulations, while maintaining a good parallel efficiency. These topics are currently under development.

Acknowledgements. This work was realized with the support of HPC@LR, a Center of Competence in High-Performance Computing from the LanguedocRoussillon region, funded by the Languedoc-Roussillon region, Europe and University Montpellier 2 Sciences et Techniques. The HPC@LR Center is equipped with an IBM hybrid Supercomputer. 


\section{References}

[1] P. Alart, D. Dureisseix, and M. Renouf. Using nonsmooth analysis for numerical simulation of contact mechanics. In Nonsmooth Mechanics and Analysis: Theoretical and Numerical Advances, volume 12 of Advances in Mechanics and Mathematics, chapter 17, pages 195-207. Kluwer Academic Press, 2005.

[2] P. Alart, D. Iceta, and D. Dureisseix. A nonlinear domain decomposition formulation with application to granular dynamics. Computer Methods in Applied Mechanics and Engineering, 205-208:59-67, 2012.

[3] P. Avery and C. Farhat. The FETI family of domain decomposition methods for inequality-constrained quadratic programming: Application to contact problems with conforming and nonconforming interfaces. Computer Methods in Applied Mechanics and Engineering, 198(21-26):16731683, 2009.

[4] M. Barboteu, P. Alart, and M. Vidrascu. A domain decomposition strategy for nonclassical frictional multi-contact problems. Computer Methods in Applied Mechanics and Engineering, 190:4785-4803, 2001.

[5] P. Breitkopf and M. Jean. Modélisation parallèle des matériaux granulaires. In 4e Colloque National en Calcul des Structures, pages 387-392, Giens, May 1999. CSMA.

[6] X. Cai, C. Farhat, and M. Sarkis. A minimum overlap restricted additive schwarz preconditioner and applications in 3d flow simulations. Contemporary Mathematics, 218:479-485, 1998.

[7] B. Cambou, M. Jean, and F. Radjaï, editors. Micromechanics of Granular Materials. ISTE Ltd. and John Wiley and Sons Inc., 2009.

[8] L. Champaney, J.-Y. Cognard, and P. Ladevèze. Modular analysis of assemblages of three-dimensional structures with unilateral contact conditions. Computers and Structures, 73:249-266, 1999.

[9] Z. Dostál, T. Kozubek, T. Brzobohatỳ, A. Markopoulos, and O. Vlach. Scalable TFETI with optional preconditioning by conjugate projector for transient frictionless contact problems of elasticity. Computer Methods in Applied Mechanics and Engineering, 247-248:37-50, 2012.

[10] Z. Dostál, T. Kozubek, A. Markopoulos, T. Brzobohatỳ, V. Vondrák, and P. Horyl. A theoretically supported scalable TFETI algorithm for the solution of multibody $3 \mathrm{~d}$ contact problems with friction. Computer Methods in Applied Mechanics and Engineering, 205:110-120, 2012.

[11] F. Dubois, M. Jean, M. Renouf, R. Mozul, A. Martin, and M. Bagneris. LMGC90. In 10e Colloque National en Calcul des Structures, Giens, 2011. CSMA.

[12] D. Dureisseix and C. Farhat. A numerically scalable domain decomposition method for the solution of frictionless contact problems. International Journal for Numerical Methods in Engineering, 50(12):2643-2666, 2001. 
[13] J. Fortin, O. Millet, and G. de Saxcé. Numerical simulation of granular materials by an improved discrete element method. International Journal for Numerical Methods in Engineering, 62:639-663, 2005.

[14] M. Gander and H. Zhao. Overlapping schwarz waveform relaxation for the heat equation in $\mathrm{n}$ dimensions. BIT Numerical Mathematics, 42(4):779 $795,2002$.

[15] W. D. Gropp, E. Lusk, and A. Skjellum. Using MPI: Portable Parallel Programming with the Message-Passing Interface. MIT Press, 1994. http://www-unix.mcs.anl.gov/mpi/.

[16] H. J. Herrmann and M. Müller. Simulations of granular materials on different scales. Computer Physics Communications, 127:120-125, 2000.

[17] T. M. P. Hoang, P. Alart, D. Dureisseix, and G. Saussine. A domain decomposition method for granular dynamics using discrete elements and application to railway ballast. Annals of Solid and Structural Mechanics, $2(2-4): 87-98,2011$.

[18] T. M. P. Hoang, G. Saussine, D. Dureisseix, and P. Alart. Domain decomposition with discrete element simulations using shared-memory parallel computing for railways applications. European Journal of Computational Mechanics, 2012. In press.

[19] D. Iceta, D. Dureisseix, and P. Alart. Mixed versus impulse-oriented domain decomposition method for granular dynamics. European Journal of Computational Mechanics, 18(5-6):429-443, 2009.

[20] M. Jean. The non-smooth contact dynamics method. Computer Methods in Applied Mechanics and Engineering, 177:235-257, 1999.

[21] F. Jourdan, P. Alart, and M. Jean. A Gauss-Seidel like algorithm to solve frictional contact problems. Computer Methods in Applied Mechanics and Engineering, 155(1-2):31-47, 1998.

[22] G. Karypis and V. Kumar. ParMETIS: Parallel graph partitioning and sparse matrix ordering. University of Minnesota. http://wwwusers.cs.umn.edu/ karypis/metis, 2003.

[23] T. Koziara and N. Bićanić. A distributed memory parallel multibody contact dynamics code. International Journal for Numerical Methods in Engineering, 87(1-5):437-456, 2011.

[24] P. Ladevèze, A. Nouy, and O. Loiseau. A multiscale computational approach for contact problems. Computer Methods in Applied Mechanics and Engineering, 191:4869-4891, 2002.

[25] P.-L. Lions. On the Schwarz alternating method. I. In First international symposium on domain decomposition methods for partial differential equations, pages 1-42, 1988.

[26] J. J. Moreau. Numerical aspects of sweeping process. Computer Methods in Applied Mechanics and Engineering, 177:329-349, 1999. 
[27] M. Renouf and P. Alart. Conjugate gradient type algorithms for frictional multi-contact problems: Applications to granular materials. Computer Methods in Applied Mechanics and Engineering, 194:2019-2041, 2004.

[28] M. Renouf, F. Dubois, and P. Alart. A parallel version of non smooth contact dynamics algorithm applied to simulation of granular medium. Journal of Computational and Applied Mathematics, 168:375-382, 2004.

[29] Z. Shojaaee, M. R. Shaebani, L. Brendel, J. Török, and D. E. Wolf. An adaptive hierarchical domain decomposition method for parallel contact dynamics simulations of granular materials. Journal of Computational Physics, 231(2):612-628, 2012.

[30] V. Visseq, A. Martin, D. Iceta, E. Azema, D. Dureisseix, and P. Alart. Dense granular dynamics analysis by a domain decomposition approach. Computational Mechanics, 49:709-723, 2012.

[31] C. Voivret, F. Radjaï, J.-Y. Delenne, and M. S. El Youssoufi. Space-filling properties of polydisperse granular media. Phys. Rev. E, 76:021301-1, 021301-12, 2007. 